\title{
Co-targeting of delta-like ligand 4 (DLL4) and vascular endothelial growth factor a (VEGF) with programmed death 1 (PD1) blockade inhibits tumor growth and facilitates anti-tumor immune responses
}

\author{
Minu Srivastava ${ }^{*}$, Christopher Murriel, Rui Yun, Erin Mayes, Hyun-Bae Jie, Fumiko Axelrod, Ming-Hong Xie, \\ Trevor Bentley, Belinda Cancilla, Raymond Tam, Gilbert O'Young, Ann Kapoun, John Lewicki, Tim Hoey, \\ Austin Gurney, Park Angie Inkyung
}

From 30th Annual Meeting and Associated Programs of the Society for Immunotherapy of Cancer (SITC 2015) National Harbor, MD, USA. 4-8 November 2015

Blocking DLL4, a Notch ligand, effectively inhibits tumor growth by increasing non-functional angiogenesis and decreasing the cancer stem cell (CSC) population. Preclinical studies have demonstrated inhibition of tumor growth by anti-DLL4 treatment and have led us to enter demcizumab, an anti-DLL4 mAb, into ongoing clinical trials. Vascular endothelial growth factor A (VEGF A) also plays a central role in inducing tumor angiogenesis. VEGF signaling is also involved in recruiting immune suppressive myeloid cells. Therefore, targeting VEGF could induce favorable immune responses against cancer. We developed a bispecific monoclonal antibody that blocks both DLL4 and VEGF which is in Phase I clinical trials. In the present study we compare the impact of anti-DLL4 in combination with anti-VEGF and anti-DLL4 in combination with anti-VEGF and antiProgrammed Cell Death Protein 1 (PD1) on anti-tumor immune responses. Our data demonstrate that the triple blockade of DLL4-VEGF-PD1 significantly inhibited tumor growth with more pronounced tumor regression. Anti-DLL4 treatment reduced IL17 production, an effect not observed with anti-PD1, blockade of DLL4-VEGF or DLL4-VEGF-PD1, suggesting that blocking DLL4 alone and together with VEGF or VEGF and PD1 might have different mechanisms for regulating immune responses. Anti-PD1 increased specific $\mathrm{CD} 8^{+} \mathrm{T}$ cell-mediated IFNgamma production while decreasing IL6. Interestingly,
IL2 was increased at the tumor site by blockade of DLL4-VEGF-PD1 compared to controls. Since IL2 is required for secondary population expansion of $\mathrm{CD}^{+}$ memory $\mathrm{T}$ cells, increased IL2 in the triple combination group suggests potential for increased $\mathrm{T}$ cell activation, maintenance and memory $\mathrm{T}$ cell function, as compared to single agent anti-DLL4 and anti-PD1. Memory CD8 ${ }^{+}$ $\mathrm{T}$ cell frequencies were increased within the total $\mathrm{CD} 8^{+}$ $\mathrm{T}$ cell population by DLL4-VEGF-PD1 triple blockade. Therefore, these results show that co-targeting of DLL4 and VEGF with PD1 might be an effective and durable anti-cancer therapy in part by promoting anti-tumor immune responses and inhibiting pro-tumor immune responses.

Published: 4 November 2015

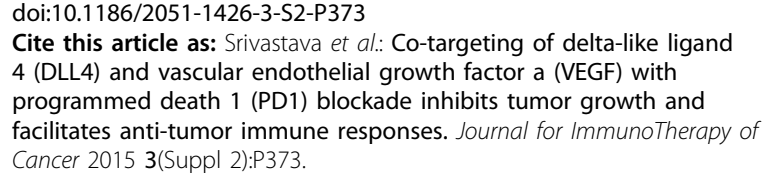

